\title{
Coexistence of the suprascapular notch and the suprascapular foramen-a rare anatomical variation and a new hypothesis on its formation based on anatomical and radiological studies
}

\author{
Michał Polguj • Kazimierz Jędrzejewski • \\ Agata Majos · Mirosław Topol
}

Received: 1 October 2012/Accepted: 17 November 2012/Published online: 4 December 2012

(C) The Author(s) 2012. This article is published with open access at Springerlink.com

\begin{abstract}
The suprascapular notch is the most common site of suprascapular nerve entrapment, which can manifest in disability and pain of the upper limb. Here, we present three cases of a very rare anatomical variation in the suprascapular region: the coexistence of the suprascapular notch and the suprascapular foramen. The variation was found during radiological and anatomical investigations. The suprascapular foramen was situated inferior to the suprascapular notch. A bony bridge lay between them, likely created by an ossified anterior coracoscapular ligament (ACSL). This anatomical variation probably increased the risk of suprascapular nerve entrapment by nerve irritation of the bony margins during passsage through the foramen and by a lack of the elasticity that the ACSL normally demonstrates. Also, a bony bridge passing through the middle part of the suprascapular notch reduces the space available for nerve passage (bony bridge decreases the space by about 36.5-38.6\%). One patient who underwent the radiological study had typical symptoms of suprascapular nerve entrapment. Based on his medical history and the presence of this rare variation of the suprascapular notch at the suprascapular region we suspect this neuropathy.
\end{abstract}

M. Polguj ( $\bowtie)$

Department of Angiology, Medical University of Łódź, Narutowicza 60, 90-136 Łódź, Poland

e-mail: michal.polguj@umed.lodz.pl

K. Jędrzejewski · M. Topol

Department of Normal and Clinical Anatomy, Medical University of Łódź, Łódź, Poland

A. Majos

Radiology Department, Medical University of Łódź, Kopcińskiego 22, 90-153 Łódź, Poland
Keywords Suprascapular foramen - Suprascapular notch . Anterior coracoscapular ligament $\cdot$ Suprascapular nerve entrapment

\section{Introduction}

The suprascapular nerve (SN) passes through the suprascapular notch (SSN) inferior to the superior transverse scapular ligament (STSL). The suprascapular region is characterised by a plurality of anatomical variations, including variations in the alignment of the nerve and vessels as well as the morphology of the bony incisura and ligaments attached to its margins. The risk of a suprascapular neuropathy can be increased by anatomical anomalies such as an ossified or bifid STSL (Duparc et al. 2010; Polguj et al. 2011), a narrow "V" shape of the suprascapular notch (Rengachary et al. 1979), a hypertrophied subscapular muscle (Duparc et al. 2010) or the presence of an anterior coracoscapular ligament (ACSL) (Avery et al. 2002; Bayramoğlu et al. 2003). The main cause of this pathological condition is a narrowing of the osteofibrous tunnel for passage of the suprascapular nerve, resulting in a mechanical irritation of the nerve during shoulder movements. Such mechanical irritation is responsible for $0.4-2 \%$ of cases of shoulder girdle pain (Gosk et al. 2007; Post and Mayer 1987). An early decompression of the nerve by resection of the STSL has proven to be a safe and effective procedure that relieves pain and restores limb function (Lafosse et al. 2007). Consequently, knowledge of these anatomical variations is of paramount importance when a decision must be made as to which procedure to use-an open, arthroscopic or arthroscopically assisted method (Urgüden et al. 2004). 


\section{Case reports}

Three unique anatomical variations at the suprascapular region were observed during radiological and anatomical studies. In all cases, a suprascapular notch coexisted with a suprascapular foramen, with the latter situated below the former, and a bony bridge lay between them. To better present this variation, we made several measurements.

\section{Collected measurements}

The following measurements of the suprascapular notch were evaluated (Fig. 1a):

1. The superior transverse diameter (STD), as the distance between the proximal and distal margins of the SSN, along the upper border of the scapula.

2. The maximal depth (MD), as the distance between the middle point of an imaginary line connected the superior corners of the notch to the deepest point of the suprascapular notch.

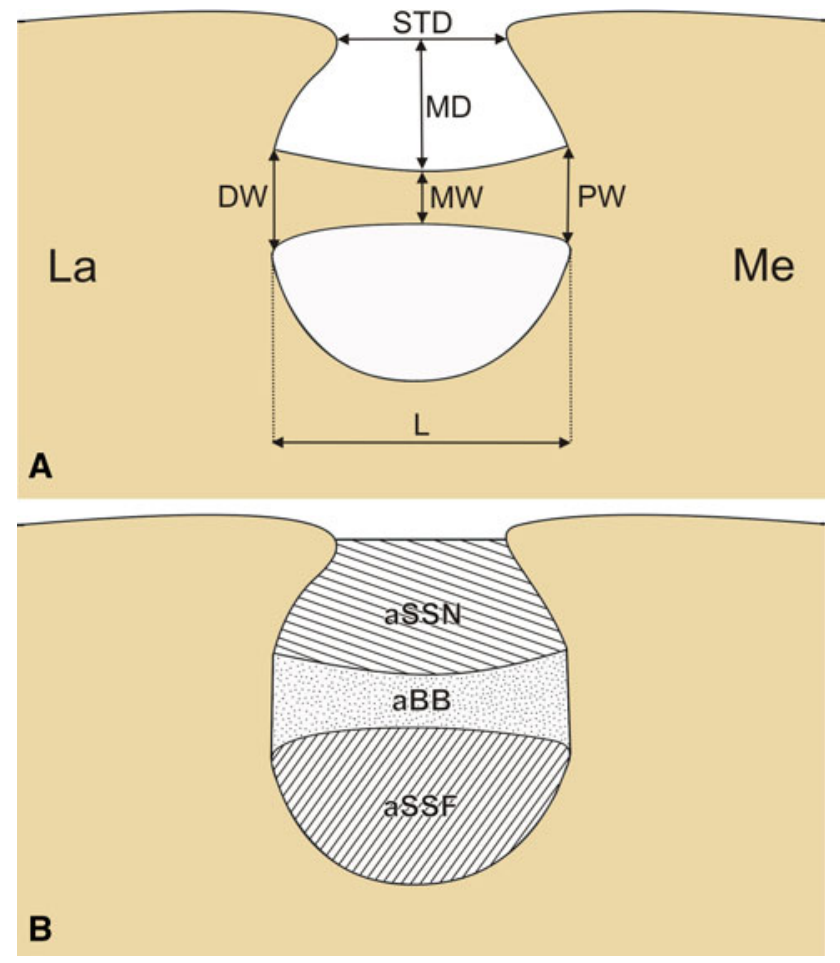

Fig. 1 Schematic representation of the arrangements of the structures in the suprascapular region of the scapula with the coexistence of a suprascapular notch and suprascapular foramen. $\mathrm{La}$ Lateral, Me medial. a Measurements of the suprascapular notch: $M D$ maximal depth, STD superior transverse diameter; measurements of the bony bridge: $L$ length, $P W$ proximal width, $M W$ middle width, $D W$ distal width. b Measurements of the areas in the suprascapular region: $a B B$ area of the bony bridge, $a S S F$ area of the suprascapular foramen, $a S S N$ area of the suprascapular notch
The following measurements of the bony bridge were collected (Fig. 1a):

1. Length (L), as the distance between proximal and distal ends of the bony bridge.

2. Proximal width (PW), as the distance between the superior and inferior borders of the bony bridge at its proximal end.

3. Middle width (MW), as the distance between the superior and inferior borders of the bony bridge half way along its length.

4. Distal width (DW), as the distance between the superior and inferior borders of the bony bridge at its distal end.

The following values were measured (Fig. 1b):

1. Area of the suprascapular foramen (aSSF)-the area limited by the osseus border of the suprascapular foramen.

2. Area of the suprascapular notch (aSSN) - the area between the superior border of the bony bridge and the superior transverse diameter (STD) of the SSN.

3. Area of the bony bridge (aBB) - the area limited by the boundaries of the bony bridge.

The following parameters were calculated using these measurements:

1. Real area for passage (raP)-the reconstructed, true area of the possible passage of the suprascapular nerve (without the area of the bony bridge)

$\mathrm{raP}=\mathrm{aSSF}+\mathrm{aSSN}$

2. Potential area for passage (paP) - the reconstructed, theoretical area for the possible passage of the suprascapular nerve

$$
\mathrm{paP}=\mathrm{aSSF}+\mathrm{aSSN}+\mathrm{aBB}
$$

3. Index reduction of passage (IrP) - a determination of the influence of the bony bridge on decreasing the potential area available for the passage the suprascapular nerve (in \%)

$$
\mathrm{IrP}=\mathrm{raP} / \mathrm{paP} \times 100 \%
$$

Radiological study

We retrospectively analyzed computed tomography (CT) scans of 308 randomised patients obtained using a standard CT chest protocol. Multidetector computed tomography (MDCT) imaging was performed with a 32-row MDCT scanner (Toshiba Aquilion 32; Toshiba Medical Systems, Otawara-shi, Tochigi, Japan). Specimens with metastases to the bone were excluded from the study. The research project and procedures were approved by the Bioethics Commission of the Medical University of Lodz (protocol 

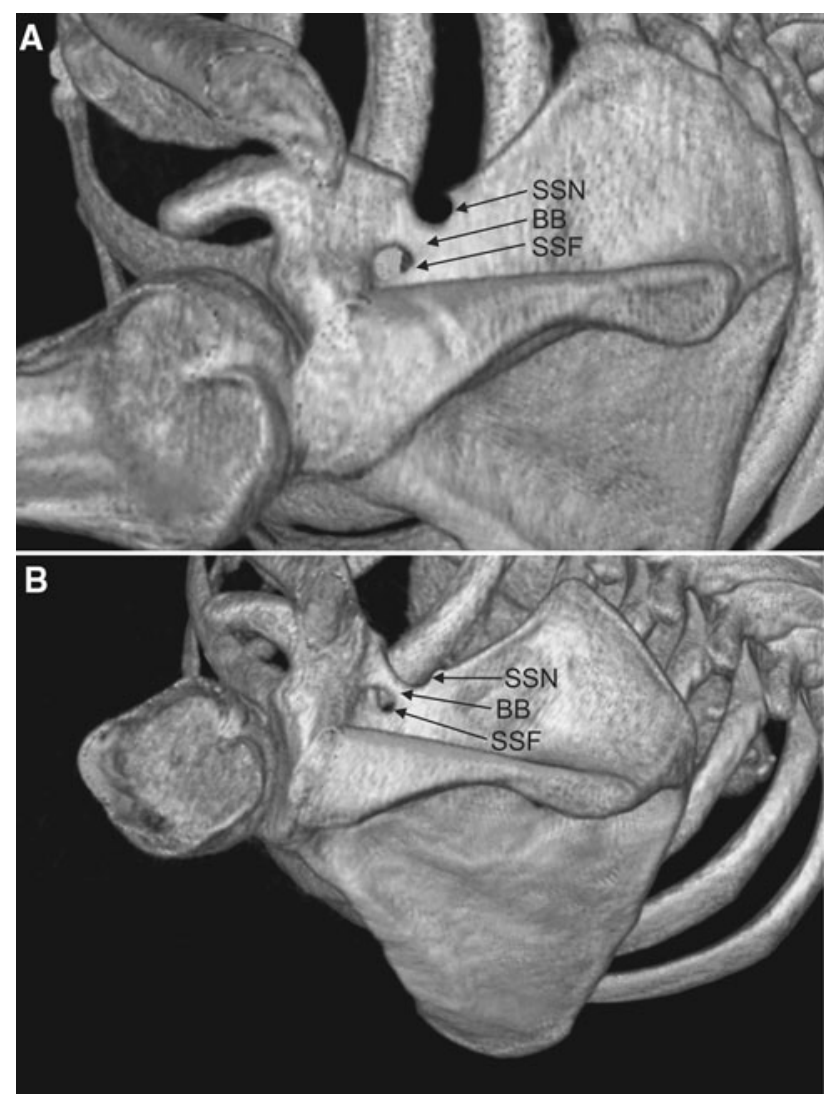

Fig. 2 Three-dimensional volume rendering (VR) multidetector computed tomography (MDCT) at suprascapular region of the scapula: $B B$ Bony bridge, $S S F$ suprascapular foramen, $S S N$ suprascapular notch. a Case 1 , b case 2

no. RNN/12/10/KE). All scapulae were analysed with postprocessing tools: multiplanar reconstructions (MPR) and maximum intensity projection (MIP) images on the coronal and sagittal planes. Coronal curved MIP images were obtained and three-dimensional (3D) volume rendering (VR) data were acquired. The measurements of the suprascapular region were performed on a 3D reconstruction of the scapula using Vitrea 2 system software (Vital Images, Plymouth, MA).

A unique anatomical variation at the suprascapular region was identified in two of the 616 randomized scapulae analysed by CT (0.33\%) (Fig. 2).

\section{Case 1}

A 41-year-old Caucasian male was admitted to the surgical department of our hospital for abdominal and chest pain associated with vomiting. A physical examination revealed palpation pain in the left epigastrium. Stomach ulcer was diagnosed by gastrofiberoscopy, and appropriate treatment was initiated. Physical examination also revealed weakness of abduction and external rotation of the left upper extremity. The patient reported suffering from deep and diffused, poorly localized pain of the left shoulder for 5 months, and further examination revealed atrophy of the left supraspinatus muscle. However, he denied even a slight trauma of this region. The right scapula had no bony bridges at the suprascapular region. The patient denied pain or weakness of the right shoulder. The right infraspinatus muscle was normal. Suprascapular neuropathy of the left shoulder was suspected based on the clinical findings.

Further examination by our scientific team identified the coexistence of a suprascapular notch and a suprascapular foramen in the left scapula. However, additional information was not available on this patient because he did not consent to an electromyographic examination to confirm the suprascapular neuropathy.

In this case of a coexisting suprascapular notch and foramen, a 11.3-mm bony bridge was present between the two structures. The width of the bridge in the proximal, middle and distal aspects was 7.2, 5.8 and $8.8 \mathrm{~mm}$, respectively. The maximum depth and superior transverse diameter of the suprascapular notch was 7.1 and $6.8 \mathrm{~mm}$, respectively. The areas of the different parts of the suprascapular notch were: aSSF, $43.4 \mathrm{~mm}^{2}$; aSSN, $40.5 \mathrm{~mm}^{2}$; aBB, $50.1 \mathrm{~mm}^{2}$. The calculated areas of the suprascapular nerve passage were: $\mathrm{raP}, 83.9 \mathrm{~mm}^{2}$; $\mathrm{paP}, 134.0 \mathrm{~mm}^{2}$, respectively (Table 1 ). The $\operatorname{IrP}$ was $37.4 \%$.

\section{Case 2}

The coexistence of a suprascapular notch and suprascapular foramen was identified in the left scapula of a 47-yearold Caucasian woman who had been hospitalised because of suspicion of pulmonary embolism (Fig. 2b). No bony bridges at the suprascapular region were observed in the contralateral scapula (right). The patient had no symptoms of suprascapular nerve entrapment syndrome. The bony bridge was $9.3 \mathrm{~mm}$ long, and its width was $6.7,4.7$ and $7.1 \mathrm{~mm}$ in the proximal, middle and distal aspects, respectively. The maximal depth and superior transverse diameter of the suprascapular notch were 5.4 and $12.8 \mathrm{~mm}$, respectively. The area of the suprascapular foramen was $39.6 \mathrm{~mm}^{2}$; the measured areas were: aSSF, $39.6 \mathrm{~mm}^{2}$; aSSN, $58.4 \mathrm{~mm}^{2}$; aBB, $61.6 \mathrm{~mm}^{2}$. The calculated areas of the suprascapular nerve passage were: $\mathrm{raP}-98.0 \mathrm{~mm}^{2}$ and paP-159.6 $\mathrm{mm}^{2}$ (Table 1). The IrP was $38.6 \%$.

Anatomical study

A search of the collection of the Department of Anatomy, Medical University of Lodz revealed a right scapula with coexisting SSN and SSF (Fig. 3). All measurements of the structures at the suprascapular region were taken using two complementary but independent methods: (1) a classical 
Table 1 Measurements of the structures at the suprascapular region in the scapula with coexisting suprascapular notch and suprascapular foramen

\begin{tabular}{|c|c|c|c|c|}
\hline \multirow[t]{2}{*}{ Structure } & \multirow[t]{2}{*}{ Measurements } & \multicolumn{2}{|c|}{$\begin{array}{l}\text { Radiological } \\
\text { study }\end{array}$} & \multirow[t]{2}{*}{$\begin{array}{l}\text { Anatomical } \\
\text { study }\end{array}$} \\
\hline & & Case 1 & Case 2 & \\
\hline \multirow{6}{*}{$\begin{array}{l}\text { Bony bridge } \\
\text { (BB) }\end{array}$} & Length (L) (mm) & 11.3 & 9.3 & 12.1 \\
\hline & Width (mm) & & & \\
\hline & Proximal (PW) & 7.2 & 6.7 & 6.4 \\
\hline & Middle (MW) & 5.8 & 4.7 & 4.6 \\
\hline & Distal (DW) & 8.8 & 7.1 & 7.9 \\
\hline & Area $\left(\mathrm{mm}^{2}\right)(\mathrm{aBB})$ & 50.1 & 61.6 & 45.1 \\
\hline $\begin{array}{l}\text { Suprascapular } \\
\text { foramen } \\
\text { (aSSF) }\end{array}$ & Area $\left(\mathrm{mm}^{2}\right)$ & 43.4 & 39.6 & 27.3 \\
\hline \multirow[t]{3}{*}{$\begin{array}{r}\text { Suprascapular } \\
\text { notch (SSN) }\end{array}$} & $\underset{(\mathrm{mm})}{\text { Maximal depth (MD) }}$ & 7.1 & 5.4 & 4.9 \\
\hline & $\begin{array}{l}\text { Superior transverse } \\
\text { diameter (STD) }(\mathrm{mm})\end{array}$ & 6.8 & 12.8 & 9.5 \\
\hline & Area $\left(\mathrm{mm}^{2}\right)(\mathrm{aSSN})$ & 40.5 & 58.4 & 51.1 \\
\hline $\begin{array}{l}\text { Real area for } \\
\text { passage (raP) }\end{array}$ & Area $\left(\mathrm{mm}^{2}\right)$ & 83.9 & 98.0 & 78.4 \\
\hline $\begin{array}{l}\text { Potential area } \\
\text { for passage } \\
\text { (paP) }\end{array}$ & Area $\left(\mathrm{mm}^{2}\right)$ & 134.0 & 159.6 & 123.5 \\
\hline
\end{tabular}

osteometry using an electronic digimatic caliper (Mitutoyo Co, Kawasaki, Japan); (2) a new method based on an analysis of digital photographic documentation of the structures at the suprascapular region using MultiScanBase v.14.02 software (Computer Scanning System II, Warsaw, Poland).

The superior transverse diameter and the maximal depth of the suprascapular notch were 9.5 and $4.9 \mathrm{~mm}$, respectively. The bony bridge was $12.1 \mathrm{~mm}$ long, and its width in the proximal, middle and distal aspects were 6.4, 4.6 and $7.9 \mathrm{~mm}$, respectively. The measured areas were: aSSF, $27.3 \mathrm{~mm}^{2}$; aSSN, $51.1 \mathrm{~mm}^{2}$; aBB, $45.1 \mathrm{~mm}^{2}$. The calculated areas of the suprascapular nerve passage were: raP, $78.4 \mathrm{~mm}^{2}$; paP, $123.5 \mathrm{~mm}^{2}$, respectively (Table 1). The IrP was $36.5 \%$.

\section{Discussion}

The patient from our first radiological study (case 1) suffered from deep and diffuse poorly localized pain of the left shoulder and weakness of abduction and external rotation of the left upper extremity in the glenohumeral joint. Atrophy of the left supraspinatus muscle was also observed. These symptoms are the main clinical manifestations of suprascapular nerve entrapment (Gosk et al. 2007; Post and Mayer 1987; Zehetgruber et al. 2002).

Although suprascapular nerve entrapment is uncommon, it should be considered in the differential diagnosis of

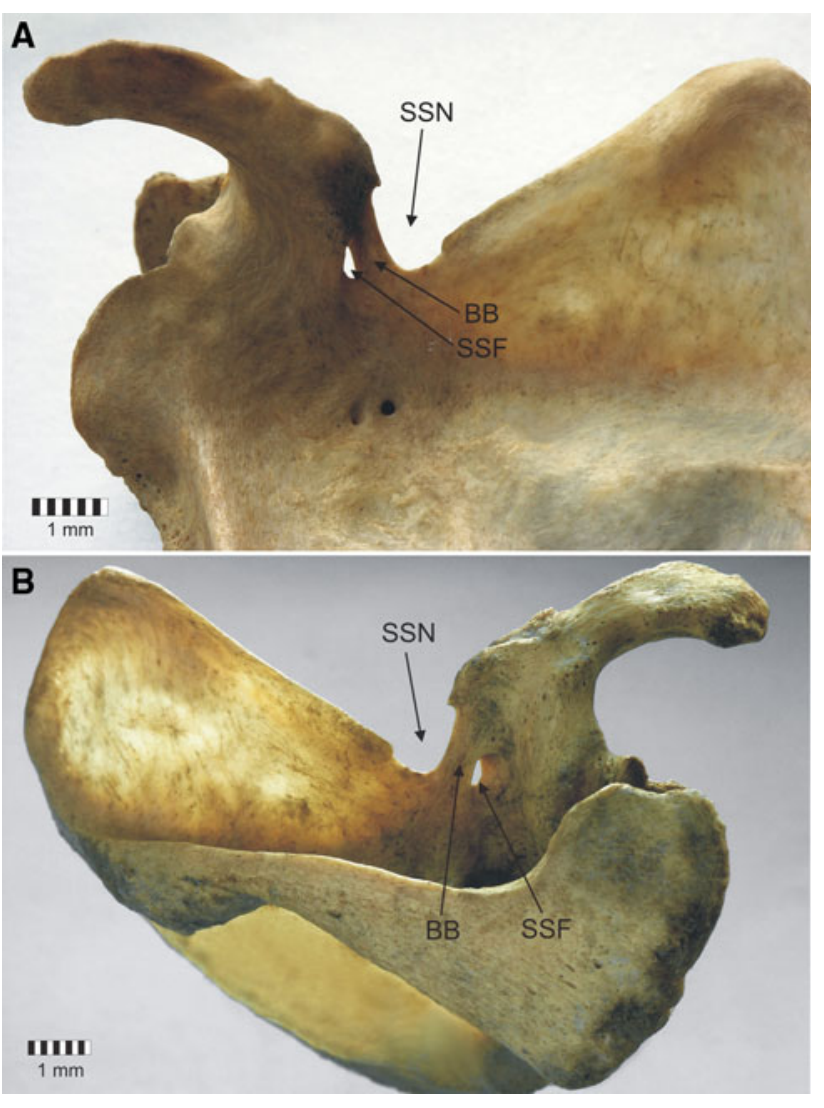

Fig. 3 Structures of the suprascapular region: $B B$ bony bridge, SSF suprascapular foramen, $S S N$ suprascapular notch. a Antero-superior aspect, $\mathbf{b}$ postero-superior aspect

patients with shoulder pain and weakness, which includes brachial plexopathy, cervical spine disease, cervical discopathy, glenohumeral joint diseases, tendonitis or bursitis and rotator cuff tear (Gosk et al. 2007; Zehetgruber et al. 2002). The diagnosis of suprascapular nerve entrapment is based on physical examination, medical history and the results of additional investigations (Cummins et al. 2000). The most important of the latter are the results from electrodiagnostic studies, including studies of nerve conduction velocity from Erb's point to the supra- and infraspinatus muscles and electromyography. The normal latency ranges for the supra- and infraspinatus muscles are 1.7-3.7 and 3.3-4.2 ms, respectively (Kraft 1972; Kullmer et al. 1998).

The patient from our radiological study (case 1) refused further diagnostic evaluation of his shoulder pain. However, based on his clinical presentation and the radiographical findings, we consider that he presented with suprascapular nerve entrapment.

Although many anatomical variants of the suprascapular notch have been reported in the literature, coexistence of the suprascapular notch and the suprascapular foramen has 
been described only three times (Hrdicka 1942; Natsis et al. 2007; Sinkeet et al. 2010). The first report was that of Hrdlicka who in 1942 found one such case among 2792 dried scapulae $(0.036 \%)$. The second report was that of Natsis et al. (2007), who discovered this rare type of anatomical variants in three of 423 scapulae from the German general population $(0.7 \%)$. Scientists have classified scapulae with both a notch and a foramen at the site of the SSN as a distinct type (Type V). More recently, there have also been descriptions from Africa; Sinkeet et al. (2010) found one such a case in 138 investigated Kenyan scapulae $(0.72 \%)$.

In our study, the frequency of the coexistence of a suprascapular notch and a suprascapular foramen was $0.33 \%$, which was higher than that reported by Hrdlicka (1942) $(0.036 \%)$ and lower than that described by Natsis et al. (2007) in German scapulae $(0.7 \%)$ and by Sinkeet et al. (2010) (0.72\%) in the Kenyan population. It would be reasonable to suppose that these frequencies depend on the population, similar to the frequency of complete ossified STSL.

To our knowledge, no hypotheses have been published to explain the coexistence of a suprascapular notch and foramen. Here, we present four hypotheses on the formation of this phenomenon which are based on the latest anatomical findings (Avery et al. 2002; Bayramoğlu et al 2003) (Fig. 4).

In our opinion, the first hypothesis (Fig. 4a) is the most probable as it assumes that the ossification of the single bundle ACSL would create the bony bridge above the suprascapular foramen. The osseous transformation of the STSL would not occur, and so it would be absent on a dry scapula, and a suprascapular notch would be formed. The ACSL was first described by Avery et al. (2002) as a fibrous band extending along the anterior aspect of the SSN, just below the STSL. Its proximal and distal attachments insert separately onto the borders of the SSN and run parallel or obliquely to the STSL. The topography of the bony bridge observed in our anatomical and radiological study was similar to that observed by Avery et al. (2002) on the anterior coracoscapular ligament. Therefore, the first hypothesis is the most probable. In our opinion, the ossification of the ACSL can increase the risk of suprascapular neuropathy because of the higher potentiality for nerve irritation by the bony margins of the foramen and the lack of elasticity that the ACSL normally demonstrates. The
Fig. 4 Schematic arrangements demonstrating four hypotheses (a-d) explaining the formation of coexisting suprascapular notch and foramen. STSL Superior transverse scapular ligament, sbSTSL superior band of superior transverse scapular ligament, mbSTSL middle band of superior transverse scapular ligament, ibSTSL inferior band of superior transverse scapular ligament, ACSL anterior coracoscapular ligament, sbACSL superior band of anterior coracoscapular ligament, $i b A C S L$ inferior band of anterior coracoscapular ligament

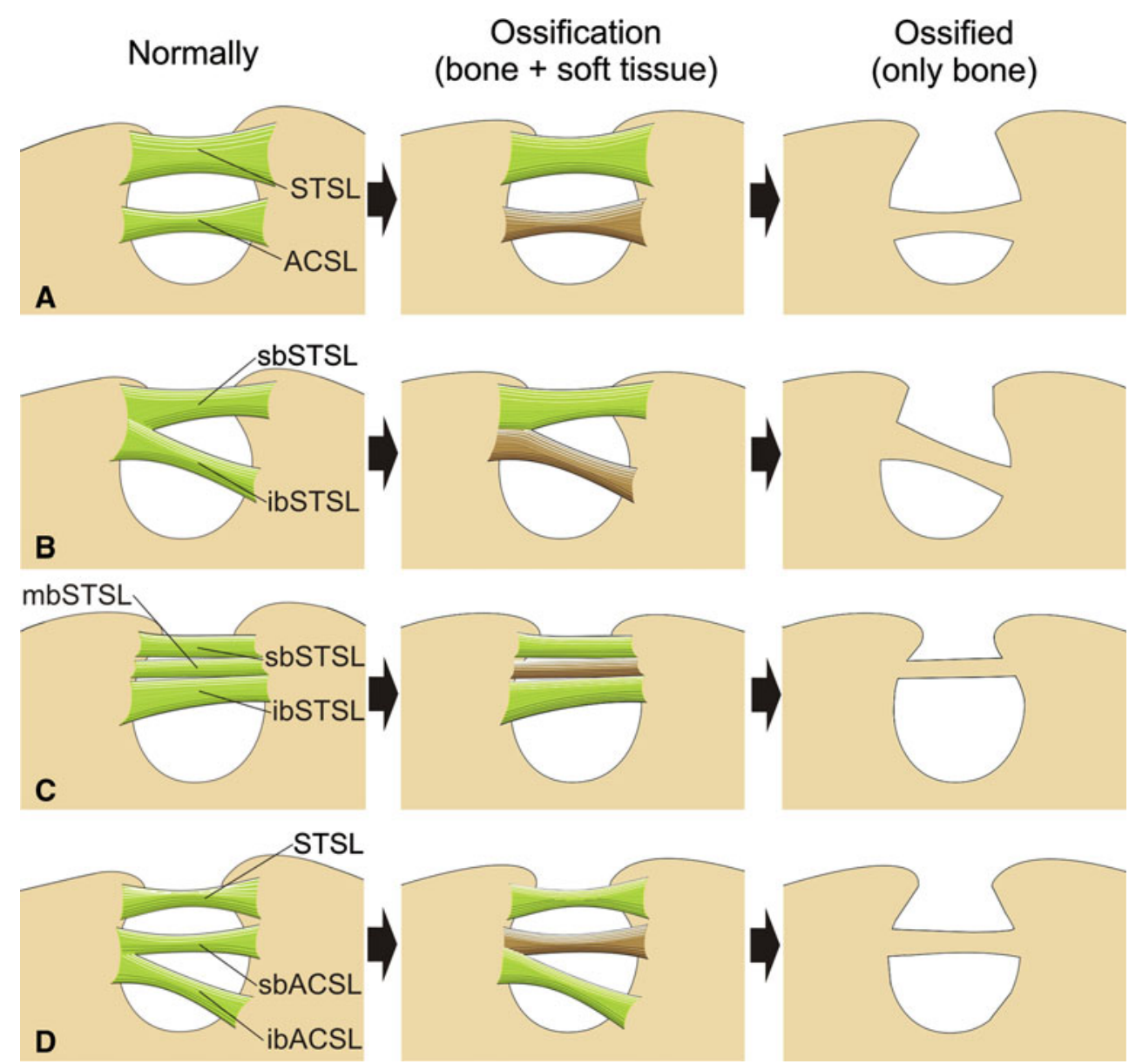


etiopathology of the suprascapular nerve entrapment proposed by Rengachary et al. (1979), called the "sling effect", assumes that during motions of the arm, the nerve makes minimal transitional movements. Therefore, an angulated nerve can be pressed against the sharp bony margin when travelling through the suprascapular foramen during the action of the upper limb. The repeated kinking irritates the nerve and induces microtrauma that might result in this neuropathy. Although in the case presented here it is impossible to determine the course of the $\mathrm{SN}$, the area of the suprascapular region was highly reduced in comparison to the potential area of the normal notch without a bony bridge. The frequency of the ACSL has been found to be $18.8 \%$ (Bayramoglu et al. 2003-Turkish population), $28 \%$ (Piyawinijwong and Tantipoon 2012Thai population) or $60 \%$ (Avery et al. 2002-American population). All scientists confirm its presence as an additional etiological factor of the condition.

The second potential mechanism (Fig. 4b) described in this article which explains the variation is calcification of the inferior part of a bifid STSL. In such a case, the ligament has two bands (superior and inferior) that are separately fixed to the one border of the suprascapular notch. Both parts of this bifid STSL travel independently one below the other, but they have a common opposing attachment. An ossified inferior band of the bifid STSL might create a bony bridge (Fig. 4b). Despite the fact that frequency of the bifid STSL varies from 3.3 to $15.6 \%$ (Bayramoglu et al. 2003; Duparc et al. 2010), partial ossification (not whole) of the ligament seems unlikely.

The third hypothesis (Fig. 4c) explaining the coexistence of a suprascapular notch and foramen depends on incomplete ossification of a trifid STSL. This type of superior transverse scapular ligament has three parts: the superior, middle and inferior band. When only the middle band ossifies, the bony bridge is formed, and the suprascapular notch and foramen are present on dry scapulae (Fig. 4c). A survey of recent literature revealed only two cases of trifid STSL (Polguj et al. 2012; Ticker et al. 1998). In the study of Ticker et al. (1998), the ligament had the middle band partially ossified, possibly confirming this hypothesis. However, Polguj et al. (2012) did not find any signs of ossification in the ligament in their study.

The fourth potential mechanism explaining the coexistence of a suprascapular notch and foramen is the calcification of the superior band of a bifid ACSL (Fig. 4d). However, this explanation seems rather unlikely because, to our knowledge, no case of partially or completely ossified bifid ACSL has ever been reported.

The SSF and the SSN are the most probable passages for the SN. Data obtained from our photographic documentation analysis indicate that the areas of both spaces were considerably decreased by the presence of the bony bridge in comparison to the reconstructed potential area of the suprascapular nerve passage. The space available for the passage was decreased by about 37.4-38.6 (radiological study) and $36.5 \%$ (anatomical study). In our opinion, the coexistence of the suprascapular notch and suprascapular foramen narrows the space for passage of the nerve and, therefore, might also increase the risk of suprascapular neuropathy.

Conflict of interest The authors declare that they have no conflict of interest.

Open Access This article is distributed under the terms of the Creative Commons Attribution License which permits any use, distribution, and reproduction in any medium, provided the original author(s) and the source are credited.

\section{References}

Avery BW, Pilon FM, Barclay JK (2002) Anterior coracoscapular ligament and suprascapular nerve entrapment. Clin Anat 15:383-386

Bayramoğlu A, Demiryürek D, Tüccar E, Erbil M, Aldur MM, Tetik O, Doral MN (2003) Variations in anatomy at the suprascapular notch possibly causing suprascapular nerve entrapment: an anatomical study. Knee Surg Sports Traumatol Arthrosc 11:393-398

Cummins CA, Messer TM, Nuber GW (2000) Suprascapular nerve entrapment. J Bone Joint Surg Am 82:415-424

Duparc F, Coquerel D, Ozeel J, Noyon M, Gerometta A, Michot C (2010) Anatomical basis of the suprascapular nerve entrapment, and clinical relevance of the supraspinatus fascia. Surg Radiol Anat 32:277-284

Gosk J, Urban M, Rutowski R (2007) Entrapment of the suprascapular nerve: anatomy, etiology, diagnosis, treatment. Ortop Traumatol Rehabil 9:68-74

Hrdicka A (1942) The adult scapula: additional observations and measurements. Am J Phys Antropol 29:363-415

Kraft GH (1972) Axillary, musculocutaneous and suprascapular nerve latency studies. Arch Phys Med Rehab 53:383-387

Kullmer K, Sievers KW, Riemers CD (1998) Changes in sonographic, magnetic resonance, tomographic, electromyographic, and histopathologic findings within a 2-month period of examinations after experimental muscle denervation. Arch Orthop Trauma Surg 117:228-234

Lafosse L, Tomasi A, Corbett S, Baier G, Willems K, Gobezie R (2007) Arthroscopic release of suprascapular nerve entrapment at the suprascapular notch: technique and preliminary results. Arthroscopy 23:34-42

Natsis K, Totlis T, Tsikaras P, Appell HJ, Skandalakis P, Koebke J (2007) Proposal for classification of the suprascapular notch: a study on 423 dried scapulas. Clin Anat 20:135-139

Piyawinijwong S, Tantipoon P (2012) The anterior coracoscapular ligament in Thais: possible etiological factor of suprascapular nerve entrapment. Siriraj Med J 64:S12-S14

Polguj M, Jędrzejewski K, Podgórski M, Topol M (2011) Morphometric study of the suprascapular notch: proposal of classification. Surg Radiol Anat 32:175-179

Polguj M, Jędrzejewski K, Majos A, Topol M (2012) The trifid superior transverse scapular ligament: a case report and review of the literature. Folia Morph (Warsz) 71:118-120 
Post M, Mayer J (1987) Suprascapular nerve entrapment: diagnosis and treatment. Clin Orthop 223:126-136

Rengachary SS, Burr D, Lucas S, Hassanei KM, Moh MP, Matzk H (1979) Suprascapular entrapment neuropathy: a clinical, anatomical, and comparative study. Part 2: anatomical study. Neurosurgery 5:447-451

Sinkeet SR, Awori KO, Odula PO, Ogeng'o JA, Mwachaka PM (2010) The suprascapular notch: its morphology and distance from the glenoid cavity in a Kenyan population. Folia Morph (Warsz) 69:241-245
Ticker JB, Djurasovic M, Strauch RJ, April EW, Pollock RG, Flatow EL, Bigliani LU (1998) The incidence of ganglion cysts and other variations in anatomy along the course of the suprascapular nerve. J Shoulder Elbow Surg 7:472-478

Urgüden M, Ozdemir H, Dönmez B, Bilbaşa H, Oğuz N (2004) Is there any effect of suprascapular notch type in iatrogenic suprascapular nerve lesions? An anatomical study. Knee Surg Sports Traumatol Arthrosc 12:241-245

Zehetgruber H, Noske H, Lang T, Wurnig C (2002) Suprascapular nerve entrapment. A meta-analysis. Int Orthop 26:339-343 